\title{
Observation of 13 mutual events of Jovian satellites performed at Lille Observatory
}

\author{
A. Vienne ${ }^{1}$, B. Noyelles ${ }^{1}$, and A. Amossé ${ }^{2}$ \\ ${ }^{1}$ Laboratoire d'Astronomie de Lille/Université de Lille, 1 impasse de l'Observatoire 59000 Lille, France \\ and Institut de Mécanique Céleste et de Calcul des Ephémérides, UMR 8028 du CNRS \\ ${ }^{2}$ Forum des Sciences, 1 place de l'Hôtel de Ville, 59650 Villeneuve d'Ascq, France
}

Received 2 June 2003 / Accepted 29 July 2003

\begin{abstract}
We have observed the four Galilean satellites of Jupiter during their mutual occultations and eclipses from February to April 2003 using a CCD camera attached to the $32.5 \mathrm{~cm}$ refractor of the observatory of Lille. We have recorded 13 lightcurves of these events. We have performed a first astrometric reduction based on the method developed in Noyelles et al. (2003). This analysis of the results and comparison with theory show that the observations are good; the residuals are about 0.03 arcsec. The observations are available in electronic form at the CDS via anonymous ftp to cdsarc.u-strasbg.fr (130.79.128.5) or via http://cdsweb.u-strasbg.fr/cgi-bin/qcat?J/A+A/410/343.
\end{abstract}

Key words. planets and satellites: individual: Jupiter - astrometry

\section{Introduction}

Observations of mutual eclipses and occultations of planetary satellites are very useful for studies on the dynamics of the satellites (see for instance Lainey et al. 2001 and Vienne \& Duriez 1995). In 2002-2003, the Sun and the Earth pass through the equatorial plane of Jupiter. A campaign of observations has been organized and the events have been predicted (Arlot 2002). The lightcurves presented in this paper are the first of this campaign.

In Noyelles et al. (2003), hereafter called Paper I, we have developed a method for reducing the lightcurves from mutual event observations. With this method, we have obtained astrometric results from the 65 events of the Saturnian system in 1995-1996. Here, we have used our experience in such reduction in order to test the quality of the observations we have obtained.

We describe the observations made and the instrumentation used. Then, a reduction is performed in order to give astrometric coordinates directly usable to reduce ephemerides and for any other dynamical studies. The details of the method of reduction are given in Paper I.

\section{The instrumentation and the observations}

All observations were made on the $32.5 \mathrm{~cm}$ refractor of Lille Observatory in France. The characteristics of the instrument and of the CCD camera we have mounted are given in Table 1.

Send offprint requests to: A. Vienne,

e-mail: Alain.Vienne@imcce.fr
Table 1. Characteristics of the instruments.

\begin{tabular}{lr}
\hline \hline diameter & $32.5 \mathrm{~cm}$ \\
focal lengh & $6 \mathrm{~m}$ \\
longitude & $0 \mathrm{~h} 12 \mathrm{~m} 17 \mathrm{~s} \mathrm{E}$ \\
latitude & $50^{\circ} 36^{\prime} 57^{\prime \prime} \mathrm{N}$ \\
altitude & $32 \mathrm{~m}$ \\
receptor & camera CCD HiSys $22,14 \mathrm{bits}$ \\
pixel & $0^{\prime} \cdot 3$ \\
field & 176 pix $\times 176 \mathrm{pix}$ \\
\hline
\end{tabular}

The weather and the availability of the instrument allowed us to record lightcurves of 13 mutual events between February 3rd and April 19th, 2003. These 3 occultations and 10 eclipses are presented in Table 2 and in Fig. 1.

The photometry has been done in a classical way. Each night, we registered some frames with the obturator closed and the same integration time as the frames of the events. This dark current frame was then subtracted from each frame of the event. We also removed the luminosity of the sky by background estimation. The lightcurves were obtained using aperture photometry. In these curves, we reported the relative flux as 1 outside the event. These flux measurements imply two satellites in cases of occultation and only the eclipsed satellite in other cases. Sometimes, it was necessary to take into account the variation of the transparency of the sky by canceling the slope of the curve outside the event. Nevertheless, this is insufficient when brutal variations of the transparency occur (see events of March 15th and March 24th).

The flux drop and the midlight time of Table 2 were found by fitting the central part of each curve to a polynomial of 

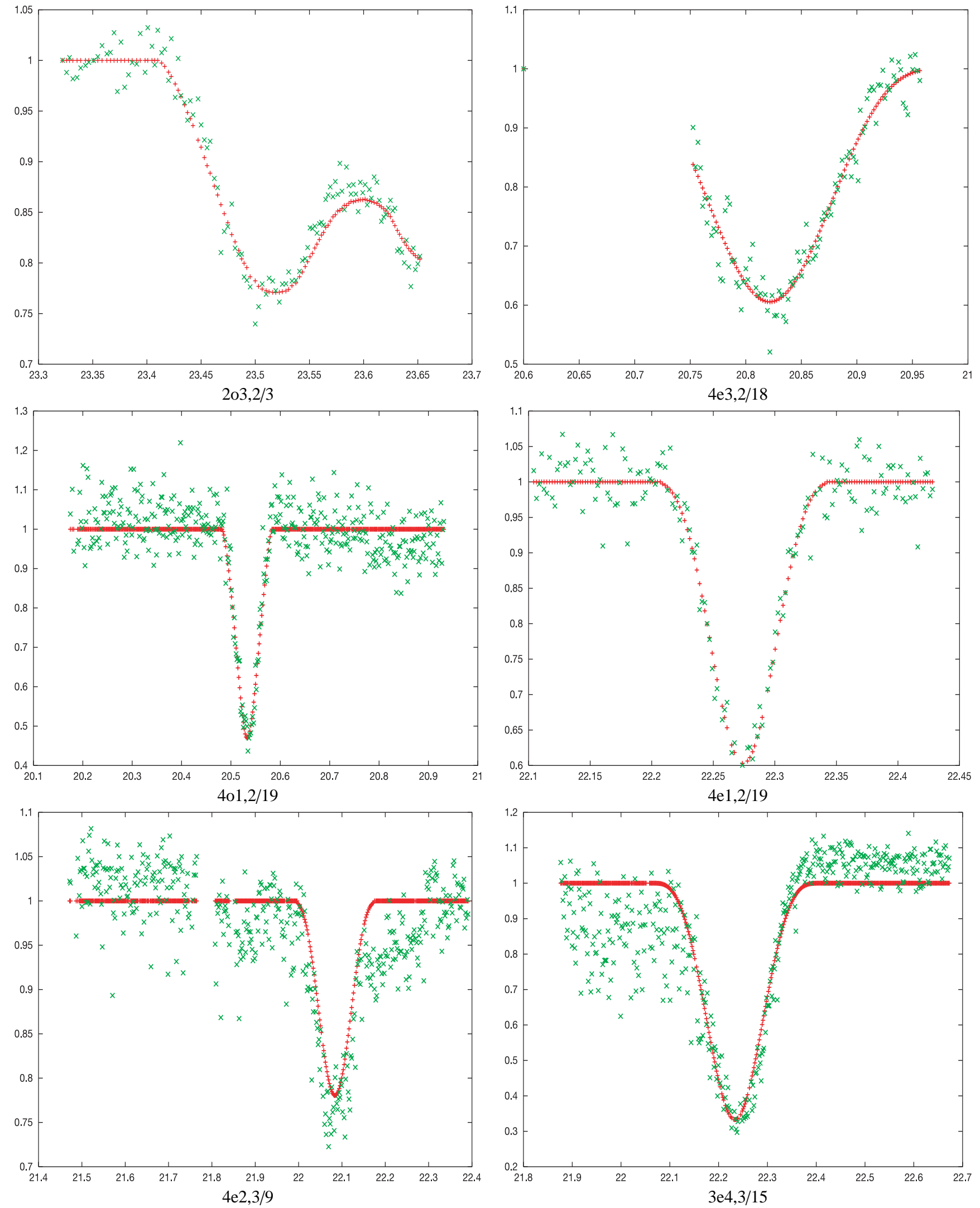

Fig. 1. The lightcurves and the fitted models. The $x$-axis corresponds to the date (in hours) and the $y$-axis to the relative flux.

degree 2. But we have to remember that the date of the minimum of distance (midtime), which is different from the midlight time, can only be given after a complete reduction, presented in the next section. Thus, in Table 2 the midlight time and the flux drop are only approximate. 

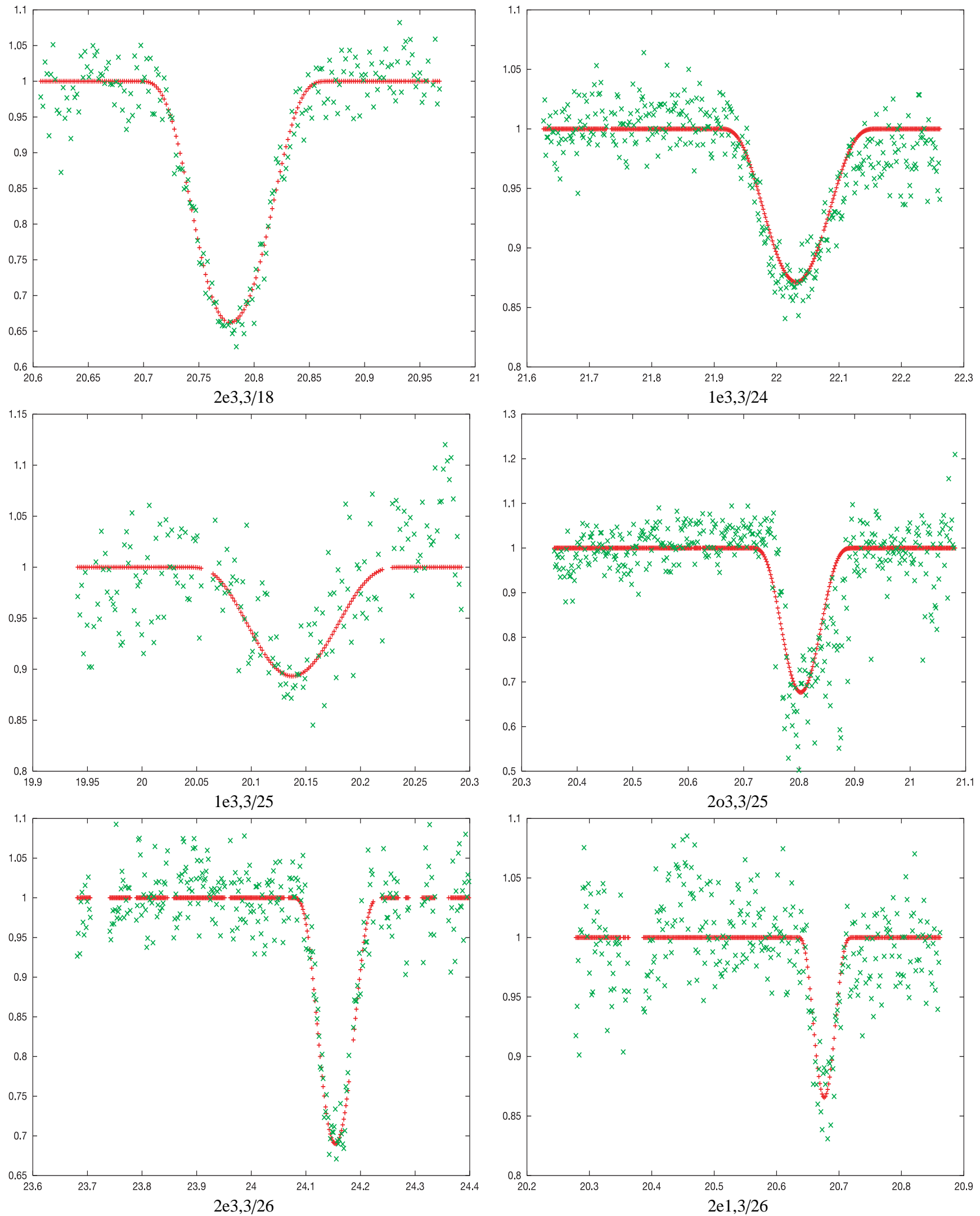

Fig. 1. continued.

The first event of February 3rd is a double one. There was, first, an occultation of Ganymede by Europa, and then, an eclipse involving the same satellites. Unfortunately, only the first event was completly recorded. Clouds covered the field during the eclipse. On February 18th we began the record after the beginning of the event because of a misunderstanding 


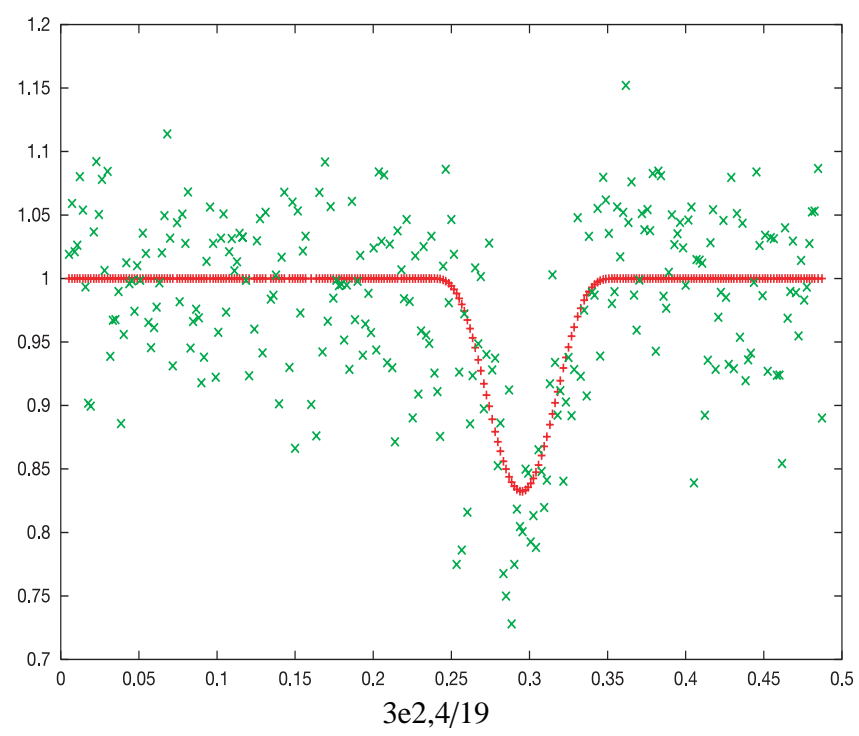

Fig. 1. continued.

Table 2. The observed mutual events. The dates are in 2003 and UTC. " $n b$ " is the number of frames and $\Delta t$ is the integration time. $\mathrm{J} 1$ refers to Io, J2 to Europa, J3 to Ganymede, and J4 to Callisto. 203 is an occultation of $\mathrm{J} 3$ by $\mathrm{J} 2$.

\begin{tabular}{cccccc}
\hline \hline event & date & $\begin{array}{c}\text { midlight time } \\
\text { h mn s }\end{array}$ & $\begin{array}{c}\text { flux } \\
\text { drop \% }\end{array}$ & $n b$ & $\begin{array}{c}\Delta t \\
\mathrm{~s}\end{array}$ \\
\hline 203 & $02 / 03$ & $233050 \pm 1$ & $23 \pm 4$ & 120 & 0.5 \\
4e3 & $02 / 18$ & $204822 \pm 5$ & $39 \pm 3$ & 99 & 1 \\
4o1 & $02 / 19$ & $203111 \pm 5$ & $52 \pm 9$ & 393 & 0.1 \\
$4 \mathrm{e} 1$ & $02 / 19$ & $221525 \pm 5$ & $37 \pm 4$ & 154 & 1 \\
$4 \mathrm{e} 2$ & $03 / 09$ & $220425 \pm 2$ & $26 \pm 8$ & 432 & 0.8 \\
3e4 & $03 / 15$ & $221425 \pm 1$ & $67 \pm 9$ & 419 & 0.5 \\
2e3 & $03 / 18$ & $204646 \pm 1$ & $35 \pm 4$ & 198 & 0.5 \\
$1 \mathrm{e} 3$ & $03 / 24$ & $220131 \pm 1$ & $13 \pm 4$ & 353 & 0.5 \\
$1 \mathrm{e} 3$ & $03 / 25$ & $200812 \pm 1$ & $11 \pm 9$ & 188 & 0.5 \\
$2 \mathrm{o} 3$ & $03 / 25$ & $204743 \pm 1$ & $35 \pm 15$ & 399 & 0.5 \\
$2 \mathrm{e} 3$ & $03 / 26$ & $000914 \pm 1$ & $31 \pm 5$ & 300 & 1 \\
$2 \mathrm{e} 1$ & $03 / 26$ & $204035 \pm 1$ & $12 \pm 7$ & 311 & 0.5 \\
3e 2 & $04 / 19$ & $001738 \pm 1$ & $16 \pm 12$ & 270 & 0.5 \\
\hline
\end{tabular}

about the prediction of its duration. Nevertheless, this event was rather well reduced.

Note that the field of the receptor $\left(52^{\prime \prime} 8 \times 52\right.$.' 8$)$ did not often allow us to have a reference satellite, or any other reference object, in the frame.

\section{First astrometric reduction}

To check the reliability of our observations, we used our experience in astrometric reduction of mutual phenomena (see Paper I) to make a first astrometric reduction of the events we observed. The method is similar to the one used in Paper I, the only differences being the use of ephemerides E5 (see Lieske 1998) to model the motion of the Galilean satellites, and the Minnaert law (see Minnaert 1961) for their photometry. We adopt this law because the photometric parameters in the Buratti-Veverka law are not well known and would be difficult to determine since their decorrelation is not guaranteed.

The Minnaert law is expressed by Eq. (1)

$\frac{I}{F}(i, e, \alpha)=B_{0}(\alpha)(\cos (i))^{k(\alpha)}(\cos (e))^{k(\alpha)-1}$

with : $\begin{cases}I & \text { reflected light per surface unit } \\ \pi F & \text { incident solar flux } \\ i & \text { light incidence angle } \\ e & \text { light emergence angle } \\ \alpha & \text { phase angle } \\ k & \text { limb darkening } \\ B_{0} & \text { photometric parameter. }\end{cases}$

The midtime corresponds to the time when the two satellites are closest on the celestial sphere. This time is slightly different to the midlight time because of light scattering by the surface of atmosphereless satellites and the phase effect. At the midtime, the impact parameter is the distance between the centre of the satellite nearer the observer and the line joining the observer to the centre of the other satellite. In the case of an eclipse, the impact parameter and the midtime are considered from the Sun's centre instead of the observer.

After modeling, we made a non-linear least squares adjustment to the midtime, impact parameter, relative velocity of the satellites, geometric albedo and limb darkening. Table 3 gives the results.

No error bars are given in Table 3 since in Paper I we are not confident about the $\sigma$ given by the non-linear least squares algorithm. In fact, the error bars on the midtime cannot be less than one second because of the accuracy of time measurement. More generally, the errors could be evaluated with the residuals. The model we used is enough accurate to check whether an observation is reliable or not.

$\Delta \alpha \cos \delta$ and $\Delta \delta$ are given in the FK5 system. We showed in Paper I that the signs of these coordinates depend upon the theory used: it is the theory and not the observation that determines which side of the line from the observer (or the Sun) 
Table 3. Results after adjustment. The coordinates are heliocentric for an eclipse, and geocentric for an occultation.

\begin{tabular}{|c|c|c|c|c|c|c|c|c|c|}
\hline event & date & $\begin{array}{l}\text { midtime } \\
\text { UTC } \\
\mathrm{h} \mathrm{m} \mathrm{s}\end{array}$ & $\begin{array}{l}\text { impact } \\
\text { parameter } \\
\mathrm{km}\end{array}$ & $\begin{array}{l}\text { limb } \\
\text { darkening }\end{array}$ & $\begin{array}{l}\text { phase } \\
\text { angle } \\
\text { degree }\end{array}$ & $\begin{array}{r}\Delta \alpha \cos \delta \\
\operatorname{arcse}\end{array}$ & ond $\Delta \delta$ & $\begin{array}{r}\mathrm{O}-\mathrm{C} 1 \\
\operatorname{arcs}\end{array}$ & $\begin{array}{l}\mathrm{O}-\mathrm{C} 2 \\
\text { cond }\end{array}$ \\
\hline 203 & $02 / 03$ & 233058 & 1075.6 & 0.498 & 0.38 & 0.109 & 0.325 & -0.012 & 0.005 \\
\hline $4 \mathrm{e} 3$ & $02 / 18$ & 204827 & 2157.4 & 0.491 & 3.44 & -0.183 & -0.530 & -0.006 & 0.062 \\
\hline $4 \mathrm{o} 1$ & $02 / 19$ & 203041 & 1567.4 & 0.460 & 3.64 & -0.152 & -0.471 & -0.167 & -0.074 \\
\hline $4 \mathrm{e} 1$ & $02 / 19$ & 221528 & 2355.8 & 0.486 & 3.65 & -0.199 & -0.579 & 0.021 & -0.003 \\
\hline $4 \mathrm{e} 2$ & 03/09 & 220507 & 3058.2 & 0.657 & 6.87 & 0.259 & 0.750 & -0.004 & -0.030 \\
\hline $3 e 4$ & $03 / 15$ & 221411 & 1215.7 & 0.521 & 7.76 & 0.106 & 0.297 & -0.072 & -0.025 \\
\hline $2 \mathrm{e} 3$ & $03 / 18$ & 204656 & 844.3 & 0.514 & 8.15 & 0.073 & 0.206 & -0.019 & -0.006 \\
\hline $1 \mathrm{e} 3$ & $03 / 24$ & 220208 & 3118.2 & 0.522 & 8.88 & -0.272 & -0.760 & 0.064 & -0.012 \\
\hline $1 \mathrm{e} 3$ & $03 / 25$ & 200826 & 3302.8 & 0.474 & 8.99 & -0.289 & -0.804 & -0.025 & -0.003 \\
\hline 203 & $03 / 25$ & 204824 & 1137.0 & 0.718 & 8.99 & -0.113 & -0.374 & 0.144 & 0.389 \\
\hline $2 \mathrm{e} 3$ & $03 / 26$ & 000928 & 1206.2 & 0.478 & 9.00 & 0.105 & 0.294 & -0.037 & -0.031 \\
\hline $2 \mathrm{e} 1$ & $03 / 26$ & 204040 & 2484.7 & 0.513 & 9.10 & -0.215 & -0.606 & -0.077 & 0.000 \\
\hline $3 \mathrm{e} 2$ & $04 / 19$ & 001747 & 3450.5 & 0.619 & 10.68 & -0.305 & -0.839 & -0.031 & 0.023 \\
\hline
\end{tabular}

to the near satellite is the far satellite. In the case of Saturnian satellites (Paper I) this uncertainty could lead to an error of 200 mas. We think this problem could appear seldom in the case of Galilean satellites, since their ephemerides are more accurate. Nevertheless, we have to remember that the signs, and only the signs, of both $\Delta \alpha \cos \delta$ and $\Delta \delta$ are theory dependent.

There are very large residuals for the occultation of Ganymede by Europa on March, 25th, and the limb darkening we find for Ganymede is very far from the other values. Moreover, there were clouds in the sky during this night, so we think that the flux drop in the lightcurve corresponds more likely to a cloud than to a mutual event. We advise against using this observation. This rejected observation illustrates the reason why it is important that the observers perform an astrometric reduction.

There are other observations in which we are very confident, for instance the first one on February 3rd because the residuals are faint and the seeing was very good. Unfortunately, clouds prevented us from observing the eclipse predicted just after the occultation. We are also confident in the observations of the two eclipses of Ganymede by Europa on March 18th and 26th because the fits seem to be visually good (the lightcurves are near the fitted models).

\section{Conclusion}

From February 3rd to April 19th, we have recorded 13 lightcurves of the mutual occultations and eclipses of the four Galilean satellites of Jupiter.
In Paper I, we obtained astrometric results from the 65 events made in 1995-1996. Here, we used our experience in such reduction in order to test the quality of the observations we have obtained. Unlike the reduction of the 1995-1996 campaign, the observations were performed by ourselves. We find that for some lightcurves, it is important that the observers perform themselves an astrometric reduction. Otherwise, the risk of misinterpreting the lightcurve is great.

The astrometric reduction based on the method developed in Paper I shows the quality of the observations presented in this paper. The precision is estimated to about 0.03 arcsec. The observations are available in electronic form at the CDS.

Acknowledgements. We thank Michel Berthe for his help in preparing the observations, and Valéry Lainey for his help in computing the ephemerides.

\section{References}

Arlot, J. E. 2002, A\&A, 383, 719

Lainey, V., Vienne A., \& Duriez L. 2001, Celes. Mech., 81, 115

Lieske, J. 1998, A\&AS, 129, 205

Minnaert, M. 1961, in Planets and Satellites, ed. G. P. Kuiper, \& B. M. Middlehurst (The University of Chicago Press), 213

Noyelles, B., Vienne A., \& Descamps P. 2003, A\&A, 401, 1159

Vienne, A., \& Duriez L. 1995, A\&A, 297, 588 